\title{
A SYSTEM OF REACTION DIFFUSION EQUATIONS ARISING IN THE THEORY OF REINFORCED RANDOM WALKS
}

\author{
Howa RD A. LEVINE \\ Department of Mathematics \\ Iowa State University \\ Ames, Iowa 50011 \\ U. S. A. \\ and \\ Brian D. SleEman \\ Department of Mathematics and Computer Science \\ University of Dundee \\ Dundee, Scotland DD1-4HN \\ United Kingdom
}

\begin{abstract}
We investigate the properties of solutions of a system of chemotaxis equations arising in the theory of reinforced random walks. We show that under some circumstances, finite time blow up of solutions are possible. In other circumstances, the solutions will decay to a spatially constant solution (collapse). We also give some intuitive arguments which demonstrate the possibility of the existence of aggregation (piecewise constant) solutions.
\end{abstract}

1991 Mathematics Subject Classification. 35K50, 35M10, 35R25, 92C45.

Key words and phrases. chemotaxis, reaction-diffusion systems, reinforced random walks.

This work was suggested by a lecture given by Hans Othmer at the University of Dundee on May 31, 1994 under the auspices of the North British Symposium on Differential Equations. Levine was supported by EPSRC grant GR/K00516, NSF-DMS-9102210 and the Institute for Mathematics and its Applications while on leave from Iowa State University. Both authors thank Hans Othmer and Angela Stevens for allowing them access to their numerical work prior to publication. They also thank Heinz Otto Kreis and Hans Weinberger for a number of stimulating conversations and elucidating remarks. 
I. Introduction. In order to understand the processes of aggregation and dispersal of cells or other organisms in a biological system, one needs to understand the mechanism of communication between such cells or organisms. Cells may interact in a variety of ways. For example, there may be long range(hormonal) interactive, intermediate range interaction via the production and release of diffusible substances or short range interactions due to local modifications of the environment such as the production and release of substances which modify the extra-cellular matrix. There may even be contact interactions via surface recognition molecules or cell-to-cell exchange of low molecular weight substances via gap functions.

A particularly interesting example combining several of these interactive processes occurs in the study of fruiting bodies such as myxococcus fulvus or the dictyostelium discoideum amoeba.

Here the fruiting body cycle begins with the development of spores which germinate and develop in vegetative growth until starved of nutrients. In this latter case the vegetative growth aggregates to form a new fruiting body to start the cycle once more. This is a complicated process which is far from being completely understood.

Dispersal often involves mechanisms that may include correlations in movement. For example, the movement of an organism in response to external stimuli may include a 'taxes' dependence on flux densities, avoidance phenomena or orientation of cells. It is well accepted that dispersal in general is not simply one of random walks (i.e. Brownian motion), but rather one of correlated or reinforced random walks [D]. Consequently it is important to address the following questions:

(1) How are the microscopic details of detection of cells to stimuli and their response reflected in the macroscopic parameters of a continuous description?

(2) Is aggregation possible without long range signaling via a diffusible attractant?

In their attempt to address these questions Othmer and Stevens [OS] have developed a number of mathematical models of chemotaxis to illustrate aggregation leading (numerically) to non-constant steady states (which appear to be stable, at least numerically), blow up resulting in the formation of singularities (in finite time) and collapse or the formation of a spatially uniform steady state. In [OS], they have recorded the results of their numerical experiments.

It is the purpose of this paper to present analytical results which support their numerical observations as well as some additional numerical computations we have made.

\section{Problem formulation.}

We consider the one space dimensional version of the Othmer-Stevens model in this paper. If $P(x, t)$ is the particle density of a particular species and $W(x, t)$ is the concentration of the "active agent", then, on some interval $(0, l)$ they consider the system of equations

$$
\begin{aligned}
\frac{\partial P}{\partial t} & =D \frac{\partial}{\partial x}\left(P \frac{\partial}{\partial x}\left(\ln \left(\frac{P}{\Phi(W)}\right)\right)\right) \\
\frac{\partial W}{\partial t} & =R(P, W)
\end{aligned}
$$


where

$$
\begin{aligned}
\Phi(W) & =\left[\frac{W+\beta}{W+\gamma}\right]^{a} \\
R(P, W) & =\frac{\lambda P W}{k_{1}+W}+\frac{\gamma_{r} P}{k_{2}+P}-\mu W
\end{aligned}
$$

and where $\beta, \gamma, k_{1}, k_{2}, \lambda, \gamma_{r}, \mu, D$ are all nonnegative constants with $D, \lambda$ being strictly positive and $a \neq 0$. One imposes the single no flux boundary condition

$$
\frac{\partial}{\partial x}\left(\ln \left(\frac{P}{\Phi(W)}\right)\right)=0 \quad \text { at } \quad x=0, l
$$

as well as given initial values

$$
\begin{aligned}
P(x, 0) & =P_{0}(x) \geq 0 \\
W(x, 0) & =W_{0}(x) \text { for } 0 \leq x \leq l
\end{aligned}
$$

Since the first of equations (OS1) is parabolic in $P$ we observe that $P(x, t) \geq 0$. More importantly, perhaps is the observation that there is no diffusion term present in the second of equations (OS1). This is in contrast to the usual chemotaxic models in which diffusion of both the population density and the chemotaxic agent occurs, e. g. $[\mathrm{CP}, \mathrm{JL}]$. Here we have a situation in which there is infinite speed of propagation in $P$ and zero speed of propagation in $W$. Therefore it might be reasonable to expect some interaction of the characteristics to produce, under appropriate choices of the parameters in (OS2), solutions for which $P$ either blows up in finite time, collapses to spatially uniform constant or collapses to a piecewise constant stationary solution. (The usual regularity theory for parabolic systems of equations is not applicable here since (OS1) is strongly coupled (the first of (OS1) involves a term of the form $\left.F(P, W) W_{x x}\right)$ and there is no diffusion in the second of equations (0S1).

Writing out the first of equations (OS1) using the second we have

$$
\frac{\partial P}{\partial t}=D\left[\frac{\partial^{2} P}{\partial x^{2}}-\frac{\partial}{\partial x}\left(\frac{a(\gamma-\beta) P}{W+\gamma)(W+\beta)} \frac{\partial W}{\partial x}\right)\right]
$$

The rigorous results we obtain are for some simplified versions of (OS). We see that if $\gamma>>W>\beta$, the coefficient of $W_{x}$ is nearly $a / W$ whereas if $\beta>>W>>\gamma$ the coefficient is $-a / W$. These two extreme cases can be modeled by taking $\Phi(W)=$ $W^{-a}$ where $a>0$ or $a<0$. In particular, with this choice of $\Phi$, throughout most of our discussion, we will take $\gamma_{r}=0, R(P, W)=\lambda P W-\mu W$. We will usually take $a= \pm 1$ also.

Thus, at the outset, we shall consider the following simplified version of (OS1OS4):

$$
\begin{aligned}
P_{t} & =D\left(P_{x x}+a\left(P \frac{W_{x}}{W}\right)_{x}\right) & & \\
W_{t} & =\lambda P W-\mu W & & \text { for } 0<x<l, t>0 \\
a \frac{W_{x}}{W}+\frac{P_{x}}{P} & =0 & & \text { for } x=0, l, t>0 \\
P(x, 0) & =P_{0}(x)>0 & & \\
W(x, 0) & =W_{0}(x)>0 & & \text { for } 0 \leq x \leq l .
\end{aligned}
$$


We shall show, among other things, that with this simplification, when $a=-1$, there are solution pairs $(P, W)$ for which $P>0$ and $P$ blows up in finite time and the power spectrum converges to that of the delta function in finite time.

When $a=1$, we will construct solution pairs $(P, W)$ for which $P>0$ and $P$ collapses to a constant in infinite time but exponentially fast.

We shall also construct traveling wave solutions (for $a= \pm 1$ ) that, in the limit of vanishing diffusivity, $D$ appear to form "standing wave" step function solutions.

For reasons that will become clear shortly, we call the case $a=-1$ or $a<0$ the mixed type case and the case $a=1$ or $a>0$ the hyperbolic case. From the second of equations $(2.1)$ it follows that since $W(x, 0)>0$, then $W(x, t)>0$ for as long as the solution $(P, W)$ exists in time.

A simple scaling argument convinces us that by writing $t=l^{2} \tau / \pi^{2} D, x=x^{\prime} \ell / \pi$ and setting $\mu^{\prime}=\mu / D$ and $\lambda^{\prime}=\lambda / D$ we see that we may take $D=1$ and $l=\pi$ in (2.1). If we multiply the first of equations (2.1) by $\lambda$ we see that we may replace $P$ by $P^{\prime}=\lambda P$. Finally, if we write $W^{\prime}=\exp (\mu t) W$ we find that we may take $\mu=0$.

After having completed these rescalings, we let

$$
\psi(x, t) \equiv \ln W(x, t) .
$$

We obtain the following initial-boundary value problem for $\psi$ :

$$
\begin{aligned}
\mathfrak{L} \psi \equiv \psi_{t t}-a\left(\psi_{x} \psi_{t}\right)_{x} & =\psi_{x x t} & & \text { for } 0<x<\pi, t>0 \\
a \psi_{x} \psi_{t}+\psi_{x t} & =0 & & \text { for } x=0, \pi, t>0 \\
\psi(x, 0) & =\psi_{0}(x) & & \\
\psi_{t}(x, 0) & =P_{0}(x)=\psi_{1}(x) & & \text { for } 0 \leq x \leq \pi .
\end{aligned}
$$

The operator $\mathfrak{L}$ defined by the first of equations $(2.3)$ is a quasilinear second order differential operator. It will be hyperbolic at a point $(x, t)$ on a function $\psi$ if and only if

$$
\psi_{x}^{2}+4 a \psi_{t}>0
$$

at $(x, t)$. If the strict reverse inequality holds, we say that $\mathfrak{L}$ is elliptic at this point on $\psi$. When equality holds, we say that the point is on the parabolic line of degeneracy for $\mathfrak{L}$ on $\psi$. Since we require that $P(x, t)=\psi_{t}(x, t)>0$, we see that (2.4) holds if $a=1$ and we refer to this case as the hyperbolic case. We refer to the case $a=-1$ as the mixed type case because then the discriminant in (2.4) can change sign when $\psi_{t}>0$.

In Figure 1 we have sketched the "hodograph" plane for the operator $\mathfrak{L}$ in the mixed type case. In order to obtain the corresponding plane in the hyperbolic case, we simply reflect the plane in the $\psi_{t}=0$ axis.

We are using quotes around the word "hodograph" because the partial differential equation in (2.4) is really a third order equation. We shall sometimes refer to this plane as the "pseudo hodograph" plane. We shall call the "characteristics" in this plane, where they exist, "pseudo characteristics". Our philisophical point of view here is that $\psi_{x x t}$ is, in some sense, a damping term which does not really affect 
Figure 1. Pseudo hodograph plane for the mixed type case $a=-1$.

the overall structure of the solution except to delay the formation of the singularity in the case of blow up or to dampen oscillatory behavior in the case of collapse.

Finally, we want to consider (OS1) in the special case in which $W>\gamma>>\beta$. In particular, we consider the system

$$
\begin{aligned}
P_{t} & =D\left(P_{x x}+a\left(P \frac{W_{x}}{W^{2}}\right)_{x}\right) & & \\
W_{t} & =\lambda P W-\mu W & & \text { for } 0<x<\pi, t>0 \\
a \frac{W_{x}}{W^{2}}+\frac{P_{x}}{P} & =0 & & \text { for } x=0, \pi, t>0 \\
P(x, 0) & =P_{0}(x)>0 & & \\
W(x, 0) & =W_{0}(x)>0 & & \text { for } 0 \leq x \leq \pi .
\end{aligned}
$$

The first of these equations also results from (OS1) when we take $\Phi(W)=$ $\exp \left(-\frac{a}{W}\right)$.

III. Solutions which blow up in finite time. We show in this section that in the mixed type case, some solutions of $(2.3)$ with $P(x, t) \geq 0$ blow up in finite time. 
We compute the power spectrum of these solutions and we show that the blow up occurs on the parabolic boundary of the hyperbolic region in the "hodograph" plane.

In this context, let us look at the data used in [OS]. The authors chose $W(x, 0)=$ constant $>0$, which constant we see by a simple scaling argument, we may take as unity. They also took $P(x, 0)=1+\varepsilon \cos (2 x)$. (They actually used $[0,1]$ as their interval but this is not important for our purposes.) This motivates us to look for a solution of the first two equations in (2.3) with $a=-1$ in the form

$$
\psi=t+u
$$

Then $u$ must satisfy

$$
\begin{aligned}
u_{t t}+u_{x x}-u_{x x t} & =-\left(u_{x} u_{t}\right)_{x} & & \text { for } 0<x<\pi, t>0 \\
u_{x}\left(u_{t}+1\right) & =u_{x t} & & \text { for } x=0, \pi, t>0 .
\end{aligned}
$$

We attempt, for fixed integer $N>0$ and $c$ real, to write $u(x, t)$ in the form

$$
u(x, t)=\sum_{n=1}^{\infty} a_{n} \exp (c N n t) \cos (N n x)
$$

(Notice that this function is harmonic in the variables $(x, c t)$ for any $c \neq 0$. ) We see that the boundary condition in (3.2) will be automatically satisfied and that

$$
u_{t t}+u_{x x}-u_{x x t}=N^{2} \sum_{n=1}^{\infty} a_{n} n^{2}\left(c^{2}+N n c-1\right) \exp (c N n t) \cos (N n x)
$$

while

$$
\begin{aligned}
-\left(u_{x} u_{t}\right)_{x}= & N^{2} c \sum_{n=2}^{\infty} \sum_{k+l=n} k a_{k} l a_{l} \exp [c N(k+l) t][\sin (N k x) \cos (N l x)]_{x} \\
= & \frac{1}{2} N^{3} c \sum_{n=2}^{\infty} \sum_{k+l=n} k a_{k} l a_{l} \exp [c N(k+l) t] . \\
& \quad[(k+l) \cos (N(k+l) x)+(k-l) \cos (N(k-l) x)] \\
= & \frac{1}{2} N^{3} c \sum_{n=2}^{\infty} n\left[\sum_{k=1}^{n-1} k(n-k) a_{k} a_{n-k}\right] \operatorname{\varepsilon xp}(c N n t) \cos (N n x) .
\end{aligned}
$$

Thus we must have, for $n=1$

$$
a_{1}\left(c^{2}+N c-1\right)=0
$$

while for $n=2,3, \ldots$,

$$
n\left(c^{2}+N n c-1\right) a_{n}=\frac{1}{2} N c \sum_{k=1}^{n-1} k(n-k) a_{k} a_{n-k} .
$$


Noting that if $c^{2}=1-N c$, we may take $a_{1}$ arbitrary and then we have

$$
2 n a_{n}=\frac{1}{n-1} \sum_{k=1}^{n-1} k(n-k) a_{k} a_{n-k}
$$

If we write $a_{n}=\frac{2 \varepsilon_{n}}{n}$ and let $\varepsilon=a_{1}=\varepsilon_{1}$ we see that

$$
\varepsilon_{n}=\frac{1}{n-1} \sum_{k=1}^{n-1} \varepsilon_{k} \varepsilon_{n-k}=\varepsilon^{n}
$$

Therefore, if we take

$$
c=\frac{-N+\sqrt{N^{2}+4}}{2}=\frac{2}{N+\sqrt{N^{2}+4}}
$$

we see that

$$
\psi(x, t)=t+2 \sum_{n=1}^{\infty} \frac{1}{n} \varepsilon^{n} \exp (N n c t) \cos (N n x)
$$

solves our problem as long as $t<T(\varepsilon, N)=\frac{-l n|\varepsilon|}{N c}$ as then the series converges absolutely and uniformly on compact subsets of $[0, \pi] \times[0, T$ ) (indeed is analytic there). The series for the density is

$$
P(x, t)=\psi_{t}=1+u_{t}=1+2 N c \sum_{n=1}^{\infty} \varepsilon^{n} \exp (N n c t) \cos (N n x)
$$

which clearly diverges if and only if $t \geq T(\varepsilon, N)$. Moreover, for sufficiently small $|\varepsilon|, P(x, 0)>0$ so that $P(x, t)>0$ on the existence interval. This series can be summed. The result is

$$
P(x, t)=1+N c(w(z)+w(\bar{z}))
$$

where $w(z)=e^{z} /\left(1-e^{z}\right)$ and $z=N c t+\ln \varepsilon+i N x$. (Since $\varepsilon<0$ is allowed, we use the principle branch of the logarithm here.) Carrying out all the algebra, we find the following

$$
\begin{aligned}
& \psi(x, t)=t-\ln \left[1-2 \varepsilon e^{N c t} \cos (N x)+\varepsilon^{2} e^{2 N c t}\right] \\
& P(x, t)=\psi_{t}(x, t)=1-2 N c \varepsilon e^{N c t} \frac{\varepsilon e^{N c t}-\cos (N x)}{1-2 \varepsilon e^{N c t} \cos (N x)+\varepsilon^{2} e^{2 N c t}}
\end{aligned}
$$

with initial values

$$
\begin{aligned}
\psi(x, 0) & =-\ln \left[1-2 \varepsilon \cos (N x)+\varepsilon^{2}\right] \\
P(x, 0) & =\psi_{t}(x, 0)=1-2 N c \varepsilon \frac{\varepsilon-\cos (N x)}{1-2 \varepsilon \cos (N x)+\varepsilon^{2}} \\
& =\frac{1+\varepsilon^{2}-2 \varepsilon(1-N c) \cos (N x)+(1-2 N c) \varepsilon^{2}}{1-2 \varepsilon \cos (N x)+\varepsilon^{2}} .
\end{aligned}
$$


Of particular interest will be the trace of (3.4) in the "hodograph" plane. In order to see what this is, we set

$$
\begin{array}{rlrl}
\xi & =\psi_{x}, & & \eta=\psi_{t} \\
\tau & =\varepsilon \exp (N c t) & & y=N x \\
\Theta & =e^{(\psi-t)} &
\end{array}
$$

We find the following formulas:

$$
\begin{aligned}
\Theta & =\left[1-2 \tau \cos y+\tau^{2}\right]^{-1} \\
\xi & =-2 N \tau \Theta \sin y \\
\eta & =1-2 N c \tau(\tau-\cos y) \Theta
\end{aligned}
$$

A somewhat tedious calculation using (3.7) yields:

$$
\begin{aligned}
c^{2} \xi^{2}+(\eta-1)^{2}-(2 c N \tau)^{2} \Theta & =0 \\
\frac{1}{4}\left(\frac{\xi}{\tau N \Theta}\right)^{2}+\left(\frac{\eta-1}{2 N c \tau \Theta}+\tau\right)^{2} & =1
\end{aligned}
$$

Using the last of equations (3.7) and the first of equations (3.8) we find that

$$
\Theta=\frac{1}{1-\tau^{2}}\left[1+\frac{\eta-1}{N c}\right]=\frac{1}{(2 N \tau)^{2}}\left[\xi^{2}+\frac{(\eta-1)^{2}}{c^{2}}\right]
$$

as long as $\tau^{2}<1$. Therefore, after a little more algebra, we find that

$$
\xi^{2}+\frac{1}{c^{2}}\left(\eta-1-\frac{2 N c \tau^{2}}{1-\tau^{2}}\right)^{2}=\frac{4 N^{2} \tau^{2}}{\left(1-\tau^{2}\right)^{2}}
$$

which is the equation of an ellipse with center at

$$
\left(0,1+\frac{2 N c \tau^{2}}{1-\tau^{2}}\right)
$$

and $\eta$ intercepts

$$
\begin{aligned}
& \eta_{-}=1-\frac{2 N c|\tau|}{1+|\tau|} \\
& \eta_{+}=1+\frac{2 N c|\tau|}{1-|\tau|}
\end{aligned}
$$

Finally, from our exact solution we find that

$$
\lim _{t \rightarrow T^{-}} \frac{e^{t} P(x, t)}{W(x, t)}=2(1-N c)(1-\operatorname{sgn}(\varepsilon) \cos (N x))
$$


where $W=\exp (\psi)$.

Notice that the blow up points of our exact solution occur when $\varepsilon e^{N c T} \cos (N \bar{x})=$ 1. This means that unless $\varepsilon<0$ when $N$ is even, the blow up points will occur at $x=0, \pi$. If $\mathrm{N}$ is even and $\varepsilon<0$, the blow up points cannot occur at the ends of the interval. In any case, blowup occurs in a finite time $T=-\ln |\varepsilon| / N c$.

We are now in a position to discuss our solution and its properties in the light of the computations of Othmer and Stevens as well as our own additional computations.

In order to do this in a systematic fashion, we shall confine our discussion to the case $N=2$. Notice from the third of formulas (3.5) that if we want to compare our choice of initial values for $P(x, 0)$ with that used in [OS], we must take our choice of $\varepsilon$ to be proportional $-2 \varepsilon([O S])(1-2 c)$ in the limit of vanishing $\varepsilon[O S]$ where this latter quantity stands for the choice of $\varepsilon$ used by them. Since $1>2 c$, this means a choice of a positive perturbation coefficient by them necessitates a negative choice by us. Of course, the data for the exact solution agrees with the Othmer-Stevens data only in the limit of $\varepsilon=\varepsilon[O S]=0$. The Othmer Stevens initial data traces a degenerate ellipse in the "hodograph" plane while the initial data for the exact solution traces out a non-degenerate ellipse in that plane (set $t=0$ in (3.9) then $\tau=\varepsilon$ there.)

It is clear from the formula for the exact solution when $N=2$ and $\varepsilon<0$ that the blow up occurs exactly at the single point $\left(\frac{\pi}{2}, T\right)$ and depends logarithmicly on $|\varepsilon|$. We note the remarkable similarity between Figures 2 and 3 . We computed the solution $P(x, t)$ using the data from [0] for various values of $\varepsilon[O S]$ and observed the same (approximate) relation between the blow up time and $\varepsilon$, namely that the blow up time is proportional to $\ln |\varepsilon|$.

The null contour line of the discriminant $D(x, t) \equiv \psi_{x}^{2}-4 \psi_{t}$ was plotted for both the O-S solution (Figure 4) and for the exact solution (Figure 5). In both figures, it is apparent that the blow up is occurring on the "parabolic"boundary. This is indeed the case as one sees from (3.9). The initial values for the exact solution prescribe a small ellipse inside the region in the "hodograph" where $\mathfrak{L}$ is elliptic. As $\tau \rightarrow 1^{-}$, these ellipses form an expanding family, exactly one member of which is tangent to the parabolic line of degeneracy. As the family continues to expand, subsequent members intersect this parabola in four points. The larger $\eta$ intercept,call it $\eta_{+}$, becomes unbounded in finite time. On setting $\xi^{2}=4 \eta$ in (3.9) we see that one of the roots of the resulting quadratic becomes unbounded in finite time while the other approaches a finite limit. Indeed, calling these roots $\bar{\eta}_{ \pm}$and the $\eta$ intercepts $\eta_{ \pm}$, we have the following asymptotics:

$$
\begin{aligned}
& \eta_{+} \approx 1+\frac{2 c N}{1-|\tau|} \approx 1+\bar{\eta}_{+} \\
& \eta_{-} \approx 1-c N \approx \bar{\eta}_{-}>0
\end{aligned}
$$

for $|\tau|$ smaller than but near 1 . There are, for the values $\bar{\eta}_{ \pm}$, four corresponding values of $\bar{\xi}_{ \pm}= \pm 2 \sqrt{\bar{\eta}_{ \pm}}$.

We can rewrite (3.9) in the form

$$
\left[c^{2} \xi^{2}+(\eta-1)^{2}\right]\left(1-\tau^{2}\right)=4 N c \tau^{2}[\eta-(1-c N)] .
$$


Figure 2. Blow up of $P$ with Othmer-Stevens initial data. $(D=0.04$. See discussion following equation (3.11) for the choice of $\varepsilon$. A precise match with the exact solution is not possible.)

We see from this that for fixed $\eta>1-N c(>0)$, as $\tau \rightarrow 1^{-}, \xi^{2} \rightarrow+\infty$. This has two consequences. First, not all of the mass is concentrated at the singularity at the blow up time. Second, The characteristics in the hyperbolic region emanating from the point $\left(\xi, \xi^{2} / 4\right)$ on the parabolic boundary for which $|\xi|>2 \sqrt{1-N c}$ intersect the line $\eta=\eta_{-}$at a finite value of $\xi$. This allows us to determine the limiting behavior of the characteristics in the hyperbolic region. In particular, these characteristics do not all focus at the blow up point.

Finally, we note that if we examine the natural logarithm of the modulus of the square of the cosine coefficients of $(P(x, t)-1) / 2 N c$ we see that these logarithms are linear in $t$, their slope increases with increasing frequency $n$, and as $t \rightarrow T(\varepsilon, N)$, these converge to zero. Precisely, they are of the form $n \ln |\varepsilon|+n N c t$. This is in conformity with the data of [OS] (Figure 6).

We conclude this discussion with some additional remarks.

Remark 3.1. The blow up in this problem is due to the nonlinear term $\left(u_{x} u_{t}\right)$ in the following sense: If we compute the Fourier transform for the initial value problem for $u_{t t}+u_{x x}=0$, we find that

$$
\hat{u}(\xi, t)=\frac{1}{2|\xi|}\left(\hat{u}_{t}(\xi, t)+|\xi| \hat{u}(\xi, t)\right) e^{|\xi| t}+\frac{1}{2|\xi|}\left(-\hat{u}_{t}(\xi, t)+|\xi| \hat{u}(\xi, t)\right) e^{-|\xi| t} .
$$

If the coefficient of $e^{|\xi| t}$ decays no faster than $e^{-a|\xi|}$ for some $a>0$ as $|\xi| \rightarrow+\infty$, 
Figure 3. Blow up of $P$ for exact solution with $N=2 .(D=0.04$. $)$

the solution will blow up in a finite time $T \leq a$. That is, the $L^{2}$ norm of the solution will become unbounded in finite time.

However, if we consider instead the initial value problem for $u_{t t}+u_{x x}=u_{x x t}$, we find

$$
\begin{aligned}
\hat{u}(\xi, t)= & \frac{1}{r_{+}(\xi)-r_{-}(\xi)}\left(\hat{u}_{t}(\xi, 0)-r_{-}(\xi) \hat{u}(\xi, 0)\right) e^{r_{+}(\xi) t} \\
& +\frac{1}{r_{+}(\xi)-r_{-}(\xi)}\left(-\hat{u}_{t}(\xi, 0)+r_{+}(\xi) \hat{(} u(\xi, 0)\right) e^{r_{-}(\xi) t}
\end{aligned}
$$

where

$$
r_{ \pm}(\xi)=\frac{1}{2}\left(-\xi^{2} \pm \sqrt{\xi^{4}+4 \xi^{2}}\right) .
$$

Since $r_{-}(\xi) \rightarrow-\xi^{2}$ and $r_{+}(\xi) \rightarrow 0$ as $|\xi| \rightarrow+\infty$, the solution does not lose regularity as it does in the absence of the damping term $u_{x x t}$. That is, the solution will stay in the same smoothness class as the initial data. Therefore the finite time blow up must be caused by the nonlinearity.

Remark 3.2. If we had chosen the negative root for $c$, we would have found that the density decays exponentially fast as $t \rightarrow+\infty$ (collapses) to a constant. The impact of this observation is that if our initial density has any component in the direction of the initial density of our non-global solution, then the corresponding solution will probably blow up in finite time. The Othmer-Stevens data bear this 
Figure 4. Sign sets for the normalized discriminant $\psi_{x}^{2}-4 \psi_{t}$ for OthmerStevens initial values data. (The unscaled discriminant is $D^{2} \psi_{x}^{2}-4 D \psi_{t}$.) The normalized determinant was used in order to artificially enlarge the size of the "hyperbolic" region in the physical plane for purposes of clarity. The actual hyperbolic lobes are somewhat small but still coaless along the line $x=0.5$ at the blow up time.

out. We found this to be the case for other initial data as well which had roughly the same "shape" as $1+\varepsilon \cos (2 x)$, namely a minimum (for $\varepsilon>0$ ) and local maxima at the endpoints.

Remark 3.3. If instead of the boundary condition $\psi_{x}(\pi, t)=0$, we have the stronger condition $\psi_{x} \psi_{t}=\psi_{x t}$ there, then we have for $0 \leq \tau \leq t$

$$
\psi_{x}(\pi, t)=\psi_{x}(\pi, \tau) e^{(\psi(\pi, t)-\psi(\pi, \tau))} .
$$

This implies that if $\psi_{x}$ is nonzero at $x=\pi$ at some time. Then it is of one sign. Suppose, in addition, that the solution is elliptic on $x=\pi$. It follows that

$$
\psi_{t}(\pi, t) \geq \frac{1}{4} \psi_{x}^{2}(\pi, \tau) e^{2(\psi(\pi, t)-\psi(\pi, \tau))} .
$$

It follows from this and a quadrature that $\psi(x, t)$ must be non-global on $x=\pi$ in some finite time $T=T\left(\psi_{x}(\pi, 0)\right)$ unless it became non-global earlier on $[0, \pi)$. Thus, if the solution is global and the normal derivative does not vanish on the boundary, the solution must change type on the boundary. 
Figure 5. Sign sets for the normalized discriminant $\psi_{x}^{2}-4 \psi_{t}$ for exact solution.

Remark 3.4. Other solutions can be found in various ways. For example, if the mean value of $P(x, 0)$ were prescribed as some other value, $\bar{p}$, say, then with $\psi=\bar{p} t+u$, $u$ must satisfy

$$
\begin{aligned}
u_{t t}+\bar{p} u_{x x}-u_{x x t} & =-\left(u_{x} u_{t}\right)_{x} & & \text { for } 0<x<\pi, t>0 \\
u_{x}\left(u_{t}+\bar{p}\right) & =u_{x t} & & \text { for } \quad x=0, \pi t>0 .
\end{aligned}
$$

instead of (3.2). The values of the constant $c$ then become

$$
c=\frac{-N \pm \sqrt{N^{2}+4 \bar{p}}}{2}=\frac{2 \bar{p}}{N \pm \sqrt{N^{2}+4 \bar{p}}} .
$$

From this formula we see that even for negative mean values of the solution it is possible to have solutions which blow up in finite time if $4 \bar{p}>-N^{2}$. If this inequality fails, we will have either one real value of $c$ for which the solution decays exponentially in time or two complex solutions which decay exponentially in time.

Hans Weinberger observed that it also possible to construct a large family of solutions which are both harmonic in $(x, t)$ and blow up in finite time. For example, writing $z=x+i t, \bar{z}=x-i t=w$, we find that

$$
4 u_{z w}=-\left(\frac{\partial}{\partial z}-\frac{\partial}{\partial w}\right)\left(u_{z}^{2}-u_{w}^{2}\right) .
$$


Figure 6. Power spectrum (square of even cosine coefficients) for O-S data. (From $[\mathrm{OS}]$.)

With $u=F(z)+G(w)$. We are then led to the pair of ordinary differential equations

$$
\begin{aligned}
F^{\prime \prime \prime}(z)-2 F^{\prime}(z) F^{\prime \prime}(z) & =\theta \\
G^{\prime \prime \prime}(w)-2 G^{\prime}(w) G^{\prime \prime}(w) & =-\theta
\end{aligned}
$$

where $\theta$ is a constant. If $\theta=0$, we may take $G(w)=\overline{F(z)}$ and we find after quadrature and taking real parts that

$$
u(x, t)=\ln \left[\cos ^{2}\left(\theta_{1} x-\alpha\right)+\sinh ^{2}(\theta t+\beta)\right]
$$

for other constants $\alpha, \beta, \theta_{1}$. If we set $\alpha=\frac{m \pi}{2}, \theta_{1}=\frac{N}{2}$ with $m, N$ as integers, we find that $u_{x}=0$ at $0, \pi$ and that both $u, u_{t}$ become singular at positive $\bar{t}=-\beta / \theta_{1}$ 
Figure 7. Initial and "final" profiles for $e^{t} P / W$ for Othmer-Stevens initial data.

if $\beta<0$ and at $\bar{x}=-(2 l+m+1) \pi / N$ where $l$ is such that $\bar{x}$ is in $(0, \pi)$. One can easily check that

$$
P(x, t)=1+u_{t}>1-\frac{\theta_{1} \sinh \left(2\left(\theta_{1} t+\beta\right)\right)}{\cos ^{2}\left(\theta_{1} x-\alpha\right)+\sinh ^{2}\left(\theta_{1} t+\beta\right)}
$$

for $t<\bar{t}$. Since $\theta_{1} t+\beta<0$ for $t<$ olt, a mean value of unity is not possible.

If $\theta \neq 0$, we find that $\theta$ must be purely imaginary and

$$
u(x, t)=-2 \ln \left|\left(z-c_{1}\right)^{\frac{1}{2}} Z_{\frac{1}{3}}\left(\frac{2}{3} \sqrt{\theta}\left(z-c_{1}\right)^{\frac{3}{2}}\right)\right|
$$

a family of solutions involving Bessel functions of fractional order which do not satisfy the no flux boundary conditions $u_{x}=0$ at $x=0, \pi$ for any constants $\theta, c_{1}$.

IV Collapse. In this section, we take $a=1$ so that now instead of (3.2) we have

$$
\begin{aligned}
& u_{t t}-u_{x x}-u_{x x t}=\left(u_{x} u_{t}\right)_{x} \quad \text { for } 0<x<\pi, t>0 \\
& -u_{x}\left(u_{t}+1\right)=u_{x t} \quad \text { for } x=0, \pi t>0 .
\end{aligned}
$$

Carrying out exactly the same type of cosine series expansion as in (3.2.1) we are led, with

$$
c=c_{ \pm}=\frac{-N \pm \sqrt{N^{2}-4}}{2}
$$


Figure 8. Initial and "final" profiles for $e^{t} P / W$ for exact solution.

to the solution

$$
\begin{aligned}
& \psi(x, t)=t+\ln \left[1+2 \varepsilon e^{N c t} \cos (N x)+\varepsilon^{2} e^{2 N c t}\right] \\
& P(x, t)=\psi_{t}(x, t)=1+2 N c \varepsilon e^{N c t} \frac{\varepsilon e^{N c t}+\cos (N x)}{1-2 \varepsilon e^{N c t} \cos (N x)+\varepsilon^{2} e^{2 N c t}}
\end{aligned}
$$

with initial values

$$
\begin{aligned}
\psi(x, 0) & =\ln \left[1+2 \varepsilon \cos (N x)+\varepsilon^{2}\right] \\
P(x, 0) & =\psi_{t}(x, 0)=1+2 N c \varepsilon \frac{\varepsilon+\cos (N x)}{1+2 \varepsilon \cos (N x)+\varepsilon^{2}} \\
& =\frac{1+\varepsilon^{2}+2 \varepsilon(1+N c) \cos (N x)+(1+2 N c) \varepsilon^{2}}{1+2 \varepsilon \cos (N x)+\varepsilon^{2}} .
\end{aligned}
$$

Notice that if $N=1$, the solution is complex while if $N=2$ there is exactly one real value of $c$. In all cases, however, $\Re\left(c_{ \pm}\right)<0$ and $P(x, t)$ decays exponentially to $\bar{p}=1$. This is the case of collapse.

The trace of (4.3) in the "hodograph" plane is again an ellipse for each time. The formulas replacing (3.7), (3.9) and (3.10) are

$$
\begin{aligned}
\Theta & =\left[1+2 \tau \cos y+\tau^{2}\right]^{-1}=e^{-(\psi-t)} \\
\xi & =-2 N \tau \Theta \sin y \\
\eta & =1+2 N c \tau(\tau+\cos y) \Theta
\end{aligned}
$$




$$
\xi^{2}+\frac{1}{c^{2}}\left(\eta-1+\frac{2 N c \tau^{2}}{1-\tau^{2}}\right)^{2}=\frac{4 N^{2} \tau^{2}}{\left(1-\tau^{2}\right)^{2}}
$$

which is the equation of an ellipse with center at

$$
\left(0,1-\frac{2 N c \tau^{2}}{1-\tau^{2}}\right)
$$

and $\eta$ intercepts

$$
\begin{aligned}
& \eta_{-}=1-\frac{2 N c|\tau|}{1-|\tau|} \\
& \eta_{+}=1+\frac{2 N c|\tau|}{1+|\tau|}
\end{aligned}
$$

where it is important to remember that as $t \rightarrow+\infty, \tau \rightarrow 0$. Thus, this ellipse "collapses" to a single point.

Notice that for $\varepsilon$ close to but smaller than 1 , the lower intercept, $\eta_{-}<0$. In other words, we have collapsing solutions even with initial data partially in the "elliptic" region of the "hodograph" plane. See Figure 9. This is a further illustration of the damping effect of diffusion.

Figure 9. Pseudo hodograph plane for the hyperbolic case $a=1$. 
Remark 4.1. Notice that if we replace $a=1$ by any $a>0$ and write $\psi=\bar{p} t+u$, (4.1) becomes

$$
\begin{aligned}
u_{t t}-a \bar{p} u_{x x}-u_{x x t} & =a\left(u_{x} u_{t}\right)_{x} & & \text { for } 0<x<\pi, t>0 \\
-a u_{x}\left(u_{t}+\bar{p}\right) & =u_{x t} & & \text { for } x=0, \pi t>0 .
\end{aligned}
$$

where $\bar{p}$ is again the mean value of $P(x, 0)=\psi_{t}(x, 0)$, we find that

$$
c=c_{ \pm}=\frac{-N \pm \sqrt{N^{2}-4 a \bar{p}}}{2} .
$$

We see from this that for fixed $N$, if $a \bar{p}$ becomes large the solution leaves the real domain and becomes complex. This is one manifestation of our contention that (4.1) models the formation of shocks.

V Aggregation and shock formation. In this section we show how it might be reasonable to expect solutions of the system (OS) to possess spatially non-constant, piecewise constant "steady state" solutions of the type indicated in Figure 10. Our contention is based on two rather extensive observations.

Figure 10. Shock formation and aggregation for (OS), from [OS].

First, we shall argue that that the seeds of such shock formation are already contained in the simple hyperbolic model case $a>0$ considered in the preceding 
section in the "zero diffusion" limit if $a \rightarrow \infty, D \rightarrow 0$ in such a way that $a D=$ const.

The second argument is intended to demonstrate that system (OS1.1-OS4) changes "type" when the chemotaxic agent, $W(x, t)$, becomes large. When this type change (from elliptic to hyperbolic) occurs, the solution will "collapse" to two different values. In other words, in the second argument, the principle thesis is that (at least after a short time) the system will, under some circumstances possess solutions which try to blow up in finite time because of the onset of singularity formation reminiscent of the singularity formation of the exact solutions of Section 3, "elliptic" singularity formation. However, the structure of (OS1.1-OS4) is such that at least for some choices of parameters, the solutions of the system can "collapse" to two different constant values in two regions of the $(x, t)$ plane and in both regions, the mechanism for collapse is that of section 4, "hyperbolic" collapse.

5.1 The "zero diffusion" limit argument. We argue as follows: First, if we consider the initial value problem for $v_{t t}-v_{x x}-\delta v_{x x t}=0$ for $\delta>0$, we find that the Fourier transform of the solution is given by

$$
\begin{aligned}
\hat{v}(\xi, t)= & \frac{1}{r_{+}(\xi)-r_{-}(\xi)}\left(\hat{v}_{t}(\xi, 0)-r_{-}(\xi) \hat{v}(\xi, 0)\right) e^{r_{+}(\xi) t} \\
& +\frac{1}{r_{+}(\xi)-r_{-}(\xi)}\left(-\hat{v}_{t}(\xi, 0)+r_{+}(\xi) \hat{v}(\xi, 0)\right) e^{r_{-}(\xi) t}
\end{aligned}
$$

where

$$
r_{ \pm}(\xi)=\frac{1}{2}\left(-\delta \xi^{2} \pm \sqrt{\delta^{2} \xi^{4}-4 \xi^{2}}\right) .
$$

which tells us that the "damping" (diffusion) term $\delta v_{x x t}$ improves, in general, the regularity of the solution over that of the initial value problem for the equation $v_{t t}-v_{x x}=0$.

Now let us observe that if we set $y=\varepsilon^{-1}\left(x-x_{0}\right), \tau=\varepsilon^{-1} t$ and $u=\varepsilon^{-1} v$ where $v$ is a solution of $v_{t t}-v_{x x}-v_{x x t}=\left(v_{x} v_{t}\right)_{x}$, the first of equations (4.1). Then, if we let $\varepsilon \rightarrow 0$, formally, we are led to consider the initial value problem

$$
\begin{aligned}
u_{t t}-u_{x x} & =\left(u_{x} u_{t}\right)_{x}, & & -\infty<x<\infty, \quad t>0 \\
u(x, 0) & =u_{0}(x) & & -\infty<x<\infty \\
u_{t}(x, 0) & =u_{1}(x) & & -\infty<x<\infty .
\end{aligned}
$$

(We have returned to original variables in (5.1) for convenience.) We look for simple wave solutions of (5.1), That is, if we set $p=u_{x}, q=u_{t}$, the first of equations (5.1) becomes the first order system

$$
\begin{aligned}
& q_{t}=(p q)_{x}+p_{x} \\
& p_{t}=q_{x}
\end{aligned}
$$

We look for a solution of the form $q=F(p)-1$ so that the initial data must satisfy

$$
u_{1}(x)=F\left(u_{0}^{\prime}(x)\right)
$$


where we now have $q_{t}=(p F(p)) p_{x}$ and $q_{x}=F^{\prime}(p) p_{x}=p_{t}$. These will hold if

$$
\left(F^{\prime}(p)\right)^{2}-p F^{\prime}(p)-F(p)=0
$$

Now (5.3) is the characteristic equation for $\psi_{t t}=\left(\psi_{x} \psi_{t}\right)_{x}$, that is, for the first of equations (2.3) with $a=1$ and the "damping" term, $\psi_{x x t}$, omitted. In particular, we must have

$$
F^{\prime}(p)=\frac{1}{2}\left(p \pm \sqrt{p^{2}+4 F(p)}\right)
$$

With such "characteristic" initial data, we obtain, by the method of characteristics, the implicit solution

$$
p(x, t)=u_{0}\left(x+t F^{\prime}(p(x, t))\right)
$$

where $F(\cdot)$ is a non-constant solution of (5.4). If we set $\sigma=x+t F^{\prime}(p)$, then implicit differentiation yields

$$
p_{x}(x, t)=\frac{u_{0}^{\prime}(\sigma)}{1-t u_{0}^{\prime}(\sigma) F^{\prime \prime}(p(x, t))}
$$

Thus, if there is no damping present, shocks will form in positive finite time along those characteristics which are strictly convex, $\left(F^{\prime \prime}>0\right)$, if and only if $u_{0}^{\prime}>0$ somewhere or along strictly concave characteristics if and only if $u_{0}^{\prime}<0$ somewhere. As long as $F^{\prime}(p) \neq 0$, this result can be appropriately recast in terms of a condition on $u_{1}$ since $u_{0}^{\prime}=F^{-1}\left(u_{1}\right)$ and consequently $u_{0}^{\prime}(x) F^{\prime \prime}\left(u_{0}^{\prime}\right)$ may be rewritten in terms of $u_{1}$.

Suppose we consider the initial value problem obtained by the rescaling $y=\varepsilon x$, $\tau=\varepsilon t$ but we do not scale in $\psi$ in (2.3). Then we have

$$
\psi_{t t}=\varepsilon\left(\psi_{x x t}+a\left(\psi_{x} \psi_{t}\right)_{x}\right)
$$

We are interested in traveling wave solutions for (5.6). We write

$$
\psi(x, t)=\varphi(x+c t)=\varphi(\sigma)
$$

substitution of which into (5.6) yields, after a quadrature:

$$
\varphi^{\prime \prime}(\sigma)=-a\left(\varphi^{\prime}(\sigma)-r_{+}\right)\left(\varphi^{\prime}(\sigma)-r_{-}\right)
$$

where $r_{+}+r_{-}=c / a \varepsilon$ and $r_{+} r_{-}=-A / \varepsilon$ for some constant of integration, $A$. If $A<0$ we will have $r_{+}>r_{-}>0$. Write $r_{ \pm}=\frac{c}{\varepsilon a} m_{ \pm}$and fix $m_{ \pm}$. Let $A=-\frac{b^{2} c^{2}}{4 a^{2} \varepsilon}$. Then, $m_{ \pm}+m_{-}=1, m_{+}>m_{-}>0$ and $m_{+} m_{-}=\frac{b^{2}}{4},\left(b^{2}<1\right)$. Integrating (5.7) in the usual manner, we find that

$$
P(x, t)=\psi_{t}(x, t)=c \varphi^{\prime}(\sigma)=\frac{c^{2}}{a \varepsilon} \frac{m_{+}+B m_{-} e^{-\frac{\left(m_{+}-m_{-}\right) c \sigma}{\varepsilon}}}{1+B e^{-\frac{\left(m_{+}-m_{-}\right) c \sigma}{\varepsilon}}}
$$

Where $B>0$ is some constant of integration. We can think of the density as a wave front traveling to the left at speed $c$. (Since replacing $x$ by $-x$ in the original 
pde leaves it unchanged we may construct a traveling wave traveling to the right in a similar manner.) The jump in this wave front is

$$
[P] \equiv \lim _{\sigma \rightarrow+\infty} P(\sigma)-\lim _{\sigma \rightarrow-\infty} P(\sigma)=\frac{c^{2}\left(m_{+}-m_{-}\right)}{a \varepsilon}
$$

Let us set $\alpha=c / \varepsilon, c^{2} / \varepsilon=\beta$, with $\beta$ fixed and let $\varepsilon \rightarrow 0, c \rightarrow 0$, (and hence $\alpha \rightarrow \infty$.) Then $[P]$ is fixed while

$$
\begin{aligned}
P_{0}(\sigma) \equiv \lim _{c, \varepsilon \rightarrow 0^{+}} P(\sigma) & =\beta\left\{\begin{array}{l}
m_{+} \text {for } \sigma>0 \\
m_{-} \text {for } \sigma<0
\end{array}\right. \\
& =\beta\left[m_{+} H(\sigma)-m_{-} H(-\sigma)\right] .
\end{aligned}
$$

where $H(x)$ is the Heaviside step function, unity for $x>0$ and zero for $x<0$. We can think of $P_{0}$ as a zero diffusion limit of traveling wave solutions. Or we can think of it as an intrinsic step function solution in the $\varepsilon=0$ limit in the rescaled variables. It is a viscosity solution which has zero speed of travel but has a finite jump. We believe that these viscosity solutions are responsible for the aggregation in the full Othmer-Stevens model.

5.2 The "change of type" argument. Here we propose an explanation of the formation of piecewise non-constant solutions based upon the change of type of a partial differential equation for $W$ which is quasi-linear in its second derivatives and contains a third order "damping" term $D W_{x x t}$.

The rough idea is the following: The structure of the system is such that when $W$ is small, (but, when $\beta$ is nonzero, not too small), the system possesses an "elliptic" instability of the sort that led to the finite time blow up of some solutions of (3.1)(3.2). That is, the solution attempts to blow up in finite time and this attempt is due to the fact that the system has an elliptic structure very much like (3.1)-(3.2) when $P>\mu$ and $W(x, t)$ is not too large. Moreover, the choice of initial values taken leads to an initial boundary value problem with data at least partially in this "elliptic" region.

However, as $W$ increases, in any region where the relative gradient

$$
\frac{\nabla W}{W}=\left(\frac{W_{x}}{W}, \frac{W_{t}}{W}\right)
$$

remains bounded, the "type" changes from elliptic to hyperbolic. When this occurs, the system behaves like (4.1) for $P>\mu$. The system will also behave like (4.1) when $\beta \neq 0$, for $W$ very small and for $P<\mu)$. The solution $(P, W)$ again collapses. However, this collapse will not be spatially uniform because of the structure of the characteristics in the hyperbolic region. In particular, there are two caustics in the $(x, t)$ plane with a pair of common vertical asymptotes in that plane with the following properties: One of them permits the flux of particles from a region of low density to a region of high density while the other prohibits the reverse flow but does not allow the particle density to concentrate. There is thus collapse to two different values corresponding to these two different hyperbolic regions. 
In order to make these ideas more precise, let us consider the following version of (OS1)-(OS4).

$$
\begin{aligned}
P_{t} & =D \frac{\partial}{\partial x}\left(P_{x}-P \frac{\Phi^{\prime}(W)}{\Phi(W)} W_{x}\right) \\
W_{t} & =\left(\frac{P}{1+\mu W}-\mu\right) W .
\end{aligned}
$$

Here $\Phi$ is given in (OS2). We have done a little rescaling and taken $\gamma_{r}=0$. (For the rescaling, we have taken $\lambda / k_{1}=1, \nu=1 / k_{1}$.) It is understood that $\nu, \mu>0$. Writing

$$
A(W) \equiv \frac{W}{1+\nu W}
$$

we have

$$
P=\frac{W_{t}+\mu W}{A(W)} .
$$

Using this in the first of equations (5.9), we eliminate $\mathrm{P}$ to find

$$
\begin{gathered}
\mathfrak{L} W \equiv W_{t t}+D\left(\frac{2 A^{\prime}(W)}{A(W)}+\frac{\Phi^{\prime}(W)}{\Phi(W)}\right) W_{x} W_{x t} \\
+D\left[\left(W_{t}+\mu W\right)\left(\frac{A^{\prime}(W)}{A(W)}+\frac{\Phi^{\prime}(W)}{\Phi(W)}\right)-\mu\right] W_{x x} \\
=D W_{x x t}-D\left(W_{t}+\mu W\right) W_{x}^{2}\left[\mu\left(\frac{2 A^{\prime}(W)}{A(W)}+\frac{\Phi^{\prime}(W)}{\Phi(W)}\right)\right. \\
\left.+A(W) \frac{d}{d W}\left(\frac{A^{\prime}(W)}{A^{2}(W)}+\frac{\Phi^{\prime}(W)}{A(W) \Phi(W)}\right)\right] \\
+\left[\left(W_{t}+\mu W\right) \frac{A^{\prime}(W)}{A(W)}-\mu\right] W_{t} .
\end{gathered}
$$

We can thus consider the system (OS) as an initial-boundary value problem for $W(x . t)$. The guiding "philosophy" will be that, in the absence of the term $D W_{x x t}$, which is, after all, not much more than a strong damping term, the behavior of $W$ should be governed by the structure of the second order operator $\mathfrak{L}$. It will be convenient, in what follows to use the negative of the relative gradient, That is, we set

$$
(\xi, \eta) \equiv-\left(\frac{W_{x}}{W}, \frac{W_{t}}{W}\right) .
$$

We also introduce the following shorthand: For $i=1,2$ we write

$$
M_{i}(W)=W\left(\frac{i A^{\prime}(W)}{A(W)}+\frac{\Phi^{\prime}(W)}{\Phi(W)}\right) .
$$

Let us also set

$$
N(W)=\mu W M_{2}(W)+W^{2} A(W) \frac{d}{d W}\left[\frac{M_{1}(W)}{W A(W)}\right]
$$


which we recognize as $W^{2}$ times the coefficient of $D\left(W_{t}+\mu W\right) W_{x}^{2}$ in (5.10).

In order to properly analze (5.10), it is necessary to rewrite it in terms of the relative gradient. We need to do this in order to capture the contribution of the term $D W_{x x t} / W$ to the terms in the relative gradient which lead to the collapse of the solution.

Inspection of Figure 10 suggests that the regions where the gradient of $P$ is large or small should play a critical role in our analysis. There are two ways in which we can proceed in order to understand how $D W_{x x t} / W$ affects the overall dynamics. One procedure might be to write out (5.10) in terms of the relative gradient by means of the substitution $\psi=-\ln W$ if we wish to study the behavior of the system for small $W$. If we wish to study the behavior for large $W$, write $Z=1 / W$ and analize the resulting equation for small $Z$. In either case we have

$$
(\xi, \eta)=-\nabla W / W=\nabla Z / Z=\nabla \psi
$$

Then we find that

$$
\begin{aligned}
\frac{W_{x x}}{W} & =-\psi_{x x}+\psi_{x}^{2}=-\frac{Z_{x x}}{Z}+\frac{2 Z_{x}^{2}}{Z^{2}} \\
\frac{W_{t t}}{W} & =-\psi_{t t}+\psi_{t}^{2}=-\frac{Z_{t t}}{Z}+\frac{2 Z_{t}^{2}}{Z^{2}} \\
\frac{W_{x t}}{W} & =-\psi_{x t}+\psi_{x} \psi_{t}=-\frac{Z_{x t}}{Z}+\frac{2 Z_{x} Z_{t}}{Z^{2}}
\end{aligned}
$$

and

$$
\begin{aligned}
\frac{W_{x x t}}{W} & =-\psi_{x x t}+\psi_{t} \psi_{x x}+2 \psi_{x} \psi_{x t}-\psi_{x}^{2} \psi_{t} \\
& =-\frac{Z_{x x t}}{Z}+\frac{4 Z_{x} Z_{x t}}{Z^{2}}+\frac{2 Z_{t} Z_{x x}}{Z^{2}}-\frac{6 Z_{x}^{2} Z_{t}}{Z^{3}}
\end{aligned}
$$

Using the substitution $\psi=-\ln W$ we obtain

$$
\begin{array}{r}
\mathfrak{L} \psi=\psi_{t t}-D\left(M_{2}(W)-2\right) \psi_{x} \psi_{x t}-D\left[\left(1-M_{1}(W)\right]\left(\mu-\psi_{t}\right)\right) \psi_{x x} \\
=D \psi_{x x t}+D \psi_{x}^{2}\left(\mu-\psi_{t}\right)\left[M_{1}(W)+N(W)\right]-D \mu \psi_{x}^{2} \\
+D \psi_{x}^{2} \psi_{t}\left[1-M_{2}(W)\right]+\psi_{t}^{2}+\psi_{t}\left(\mu-\psi_{t}\right) \frac{W A^{\prime}(W)}{A(W)}
\end{array}
$$

where we have continued to call the second order operator $\mathfrak{L}$.

The operator $\mathfrak{L}$ is said to be elliptic at a point $Q=(W, \xi, \eta)$ if

$$
B(Q) \equiv D^{2}\left[M_{2}(W)-2\right]^{2} \xi^{2}+4 D\left[(\mu-\eta)\left(1-M_{1}(W)\right)\right]<0 .
$$

It is hyperbolic at $Q$ if $B(Q)>0$ and parabolic if $B(Q)=0$. At points where it is hyperbolic, the numbers

$$
r_{ \pm}=\frac{1}{2} D\left[M_{2}(W)-2\right] \xi \pm \frac{1}{2} \sqrt{B(Q)}
$$


are the characteristic directions (the negative reciprocals of the slopes of the characteristic curves given by $t=f_{ \pm}(x, c)$.)

At a point $Q$ where $W_{x}=W_{t}=0$, we have

$$
B(Q)=4 D \mu W\left[\frac{\nu}{1+\nu W}-\frac{\delta}{(W+\beta)(W+\gamma)}\right] .
$$

(Here and throughout the remainder of this paper we have set $\delta=a(\gamma-\beta)$.)

We see from this that if a regular critical point of $W$ occurs when $W$ is large, then this point will be in the hyperbolic region for $\mathfrak{L}$. On the other hand, for small $W$, it is entirely possible that a critical point can occur in a region where $\mathfrak{L}$ is elliptic since the equation (5.10) and the approximates considered below are in fact third order and the classical maximum principles do not apply. However, as we shall see below, if $\max W$ is sufficiently large, it must occur at a point in the hyperbolic region.

Suppose that $B(Q)=0$. Then, necessarily,

$$
C(Q) \equiv 4 D\left[\left(1-M_{1}(W)\right)\left(\mu-\psi_{t}\right)\right] \leq 0
$$

with strict inequality at those points where $\left(M_{2}(W)-2\right) W_{x} \neq 0$. It follows that wherever $\left[M_{2}(W)-2\right]^{2} \neq 0$ the slopes of the characteristics emanating from a point on the parabolic line of degeneracy must be nonzero and of the same algebraic sign near the line of degeneracy at those points where $\left(M_{2}(W)-2\right) W_{x} \neq 0$. On the other hand, at a point where $B(Q)>0$ and $C(Q)>0$, the slopes will be of opposite sign. (Of course when $B(Q)>0$ and $C(Q)<0$, the slopes will be of the same algebraic sign. This is illustrated in Figure 11.

Notice also that since

$$
\mu-\psi_{t}=\frac{P}{1+\nu W}>0
$$

the sign of $C(Q)$ is determined only by the sign of $1-M_{1}(W)$.

It is clear that in the $(\xi, \eta)$ plane, the vertex of the parabolic line of degeneracy always occurs at the point $(0, \mu)$ whenever the curve given by $B(Q)=0$ for fixed $W$ is a nondegenerate parabola, i.e. whenever $W$ is such that $\left(M_{1}(W)-1\right)\left(M_{2}(W)-\right.$ $2) \neq 0$. Morever, in such cases, this parbola will open downward (is concave down) if and only if $1-M_{1}(W)>0$. When this parabola opens downward, the points $(\xi, \eta)$ which lie in the "elliptic" region will correspond to values of the density $P$ which are negative and hence nonphysical.

Since

$$
1-M_{1}(W)=W\left[\frac{\nu}{1+\nu W}-\frac{\delta}{(W+\beta)(W+\gamma)}\right],
$$

this is clearly the case for $W$ sufficiently large. Moreover, if $\beta=0$, this parabola must open upward if $W$ is sufficiently small (and positive). If $\beta>0$ then this parabola will open downward for small $W$ if and only if

$$
\nu \beta \gamma>\delta
$$

The parabola always opens downward if the roots of the quadratic

$$
q(W)=W^{2}+(\beta+\gamma-\delta) W+(\beta \gamma-\delta) / \nu
$$


Figure 11. The characteristic slopes $1 / r_{+}(x, t)$ and $/ 1 / r_{-}(x, t) /$ plotted using the data in Figure 12.4 at time level $t=0.95$. The slopes of these characteristics are the numbers $1 / r_{ \pm}$rather than the negative reciprocals because the characteristics in Figure 16 below are plotted for the function $W=\exp (-\psi)$. Notice that near the center of the interval, the slopes have opposite sign while near the ends they have the same sign. Put another way, we see that to the right of $x=0.556$ transport along the normals to both characteristics is to the left while to the left of $x=0.445$, the propagation is to the right. In the interval $[x=0.445,0.556]$ material transport is to the left and to the right.

are either purely imaginary or the larger root is negative. That is, we will always be in a "hyperbolic" region whenever $\eta>\mu$ (or $W_{t}<-\mu W$ if either

$$
\nu(\beta+\gamma-\delta)^{2}<4(\beta \gamma-\delta)
$$

or

$$
\nu(\beta+\gamma-\delta)^{2} \geq 4(\beta \gamma-\delta), \text { and both } \quad \beta+\gamma-\delta>0, \quad \beta \gamma-\delta \geq 0
$$

hold. Thus, under these conditions on the constants, we should expect to have decay to a uniformly constant solution for $P(x, t)$ independently of the nonnegative density distribution and the (positive) value of $W(x, 0)$. This is illustrated in Figure 12 for condition (h1). 
Figure 12. Collapse when (h1) holds. Here $W(x, 0)=0.0025, P(x, 0)=$ $1-0.3 \cos (2 \pi x)$. Also $\mu=10.0, \nu=1 / k_{1}, \gamma_{r}=0.0, \kappa_{1}=10 \gamma=10 \delta=$ $10^{4}, D=0.036, \lambda=10^{4}, \beta=1.01$.

However, if (h1) and (h2) fail, simply forcing $W(x, 0)$ to be sufficiently large is insufficient to cause collapse. Indeed, as we see in Figures 13.n and 14.n, aggregation is possible even when the data are such that (5.10) is initially hyperbolic. In particular, we see from Figure 14.1 that although the discriminant

$$
D(x, t) \equiv B(Q) \equiv B(W(x, t), \xi(x, t), \eta(x, t))
$$

is initially positive, it becomes negative and then changes sign once more.

We have set, in Figures 14,n

$$
E(x, t) \equiv C(W(x, t), \xi(x, t), \eta(x, t)) .
$$

In the regions where $D(x, t)>0$, the slopes of the characteristics of $\mathfrak{L}$ have the same or the opposite sign according as $E<0$ or $E>0$. Of course it can happen that $E=D=0$ at points in the $(x, t)$ plane.

If $P(x, 0)>0$, the sign of $E$ is determined solely by the sign of $1-M_{1}(W)$, since then, by the maximum principle, $P(x, t)>0$, the finite difference scheme will sometimes not properly reflect the maximum principle. See the discussion of the numerics in the next section.

Notice that as we decrease $W(x, 0)$, there is a narrowing of the region of aggregation as well as an increase in the maximum value of the density. 
Figure 13.1. System attempt at aggregation formation when $W(x, 0)=$ $e^{7.3}$. Here $P(x, 0)=1-0.3 \cos (2 \pi x)$. Also $\mu=10.0, \gamma_{r}=0.0, \kappa_{1}=\gamma=$ $\delta=10^{3}, D=0.036, \lambda=10^{4}, \beta=0.01, \nu=1 / k_{1}$.

Figure 13.2. Same data as in Figure 13.1 but $W(x, 0)=e^{5}=148.4$. 
Figure 13.3. Same data as in Figure 13.2 but $W(x, 0)=e=2.718$.

Figure 13.4. Same data as in Figure 13.3 but $W(x, 0)=e^{-2}=0.1353$. 
Figure 13.5. Same data as in Figure 13.4 but $W(x, 0)=e^{-5}=0.00679$.

If we have $D(x, t)=E(x, t)=0$ along some curve in the $(x, t)$ plane, and if $D$ changes sign across this curve, then the characteristics emanating from this line of parabolic degeneracy will have directions of opposite sign. Moreover, the condition $D(x, t)=E(x, t)=0$ will in general, only occur when $M_{1}(W)=1$, in which case $W$ must be constant along such a curve, and $W_{x}=0$ along that curve (except in rare circumstances that $M_{2}(W)=2$ along this curve also. When $D(x, t)=E(x, t)=0$, the characteristics emanating from the parabolic boundary into the "hyperbolic" region will have vertical tangents at the parabolic boundary.

By analogy with Figures 1 and 9, we define

$$
E 1(x, t) \equiv-3 D^{2}\left[M_{2}(W)-2\right]^{2} \xi^{2}+4 D\left[(\mu-\eta)\left(1-M_{1}(W)\right)\right]
$$

The nodal line set for $E 1$ can be thought of as the second of the two local caustics in the "hyperbolic" region. (The first is the level set for $E(x, t)$.) These level sets play an important role in helping us to understand the local dynamics in the hyperbolic region.

For different constants but a very small initial $W(x, 0)$, the initial density will eventually start to form a singularity. See the figures below.

We next make a more detailed analysis of (5.10) over the following ranges:

a. $\beta>0$ and either $0<W<<\beta$ or $W \approx \beta$.

b. $\beta=0$ and $W<<1$.

c. $W \approx \gamma$.

d. $W>>\gamma$.

(It is somewhat easier to work with (5.10) than (5.10.1) but the results below also follow from (5.10.1) using the same asymptotic approximations.) 
Figure 14.1a. The regions of "ellipticity" $(D(x, t)<0)$ and "hyperbolicity" $(D(x, t)>0)$ for the data in Figure 13 for small times. The regions where the slopes of the characteristics have the same sign $(E(x, t)>0)$ and the opposite sign $(E(x, t)<0)$ are also shown.

We also assume $0 \leq \beta<<\gamma, W(x, 0>0$ and $0<\nu<1$.

Suppose first that $\beta>0$ and $W<<\beta$. We use the approximations

$$
\begin{aligned}
\frac{A^{\prime}(W)}{A(W)}=\frac{1}{W(1+\gamma W)} & \approx \frac{1}{W} \\
\frac{\Phi^{\prime}(W)}{\Phi(W)} & \approx \frac{\delta}{\beta \gamma}
\end{aligned}
$$

Then (5.10) reduces to

$$
W_{t t}+2 D \frac{W_{x} W_{x t}}{W}+D \frac{W_{t} W_{x x}}{W}=D W_{x x t}+\frac{W_{t}^{2}}{W}+2 D\left(\frac{W_{t}}{W}+\mu\right)\left(\frac{W_{x}}{W}\right)^{2}
$$

Using the substitution $\psi=-\ln (W)$, this reduces to

$$
\psi_{t t}=D \psi_{x x t}-D \mu \psi_{x}^{2}
$$

which is parabolic in $\psi_{t} \approx \mu-P=-W_{t} / W$. Thus, if $P>\mu$ on some subinterval of the interval $[0, \ell]$ initially, then $\psi_{t}<0$ there. But the differential equation tells us that we can expect $\psi_{t}$ to increase toward zero or $P$ to decrease toward $\mu$ for a short time on that interval. Similarly, if $P<\mu$ on some initial interval we expect $P$ to increase toward $\mu$ there. Indeed, this is what is observed numerically. 
Figure 14.1b. This is the same as Figure 13.1.a but for large times. Notice that the second caustic has separated itself from the first caustic, $E(x, t)=0$.

Now as long as $P>\mu(1+\nu W)$, we know that $W_{t}>0$ and $W$ will increase. If $W$ were to become unbounded in finite or infinite time, then, at least at some points in the space-time strip, for example along the line $x=\ell / 2$, it is reasonable to entertain the possibility that in finite time $t_{0}, W\left(\ell / 2, t_{0}\right)=\beta$. Of course, if $W$ does not become unbounded in finite or infinite time, then eventually, $P<\mu(1+\nu W)$ and $W$ should decay to a constant (collapse).

Let us consider therefore, the situation when $W \approx \beta$. Then, since $\nu \beta<<1$, we take

$$
\begin{aligned}
\frac{\Phi^{\prime}(W)}{\Phi(W)} & \approx \frac{\delta}{2 \gamma W} \\
\frac{A^{\prime}(W)}{A(W)}=\frac{1}{W(1+\gamma W)} & \approx \frac{1}{W} \\
A(W)\left[\frac{d}{d W}\left(\frac{A^{\prime}(W)}{A^{2}(W)}+\frac{\Phi^{\prime}(W)}{A(W) \Phi(W)}\right)\right] & \approx-\frac{2}{W^{2}}\left(1+\frac{\delta}{2 \gamma}\right)
\end{aligned}
$$

If we set $c=\delta / 2 \gamma$, we find

$$
\begin{aligned}
W_{t t}-\frac{W_{t}^{2}}{W}+D(2+c) \frac{W_{x} W_{x t}}{W^{2}}+ & D\left((1+c) \frac{W_{t}}{W}+\mu c\right) \frac{W_{x x}}{W} \\
= & D W_{x x t}+2 D(1+c)\left(\frac{W_{t}}{W}+\mu\right)\left(\frac{W_{x}}{W}\right) .
\end{aligned}
$$


Figure 14.2. Same data as in Figure 13.2. The separation of the caustics is now more pronounced.

Figure 14.3. Same data as in Figure 13.3. Further separation of the caustic lines. 
Figure 14.4. Same data as in Figure 13.4. Notice how the second caustic has now "collapsed."

Figure 14.5. Same data as in Figure 13.5. Notice the similarity of this figure to that of Figures 4 and 5 . We have an attempt at finite time blow up. 
Figure 15. Initial decay for small $W(x, 0)=0.0025$. Notice that the density at first tends to decay to unity and then starts to increase in the center. Here the remaining constants are as in Figure 13.4.

On setting $\psi=\ln (\beta / W)$ we obtain

$$
\mathfrak{L} \psi=\psi_{t t}-D c \psi_{x} \psi_{x t}+D c\left(\mu-\psi_{t}\right) \psi_{x x}=D \psi_{x x t}-2 D \mu \psi_{x}^{2} .
$$

Therefore, for $W \approx \beta$ we expect the local dynamics to be governed by

$$
\mathfrak{L} \psi=\psi_{t t}-D c \psi_{x} \psi_{x t}+D c\left(\mu-\psi_{t}\right) \psi_{x x}=D \psi_{x x t} .
$$

For the equations (5.12), (5.13), the discriminant becomes

$$
(D c)^{2} \psi_{x}^{2}-4 D c \frac{P}{1+\nu W}
$$

which will be negative if $\left|\psi_{x}\right|<<1$. This in turn holds near a local maximum or minimum of $\psi$ (minimum or maximum of $W$ (unless the density $P$ is also very small or $W$ is very large). When $W \approx \beta$ is small, then $\psi_{t} \approx \mu-P$. In particular, this means that near a point where $\psi_{t}$ changes sign but $\left|\psi_{x}\right|^{2}<\frac{4 P}{D c(1+\nu W)}$, we can expect the onset of blow up (elliptic instability) to occur. Moreover, when the spatial gradient is large $\left(\left|\psi_{x}\right|^{2}>\frac{4 P}{D c(1+\nu W)}\right)$ we will have "hyperbolic" stability, i. e. we may expect the solution to collapse. (Indeed, equation (5.13) possesses exact solutions which show precisely this behavior.) 
When $\beta=0$, the situation is similar. We now set $\psi=-\ln W$ and $c=\delta / \gamma$. Then for sufficiently small $W>0$, we have, instead of (5.12)

$$
\mathfrak{L} \psi=\psi_{t t}-D c \psi_{x} \psi_{x t}-D c \psi_{t} \psi_{x x}=D \psi_{x x t}-2 D \mu(1+c) \psi_{x}^{2}
$$

solutions of which should behave much like solutions of

$$
\mathfrak{L} \psi=\psi_{t t}-D c \psi_{x} \psi_{x t}-D c \psi_{t} \psi_{x x}=D \psi_{x x t} .
$$

That is, the $\beta=0$, small $W$ approximation is precisely the same as the simple model problem (2.1) with $a=1$. When we required that $\psi_{t}>0$ as we did in Section 4, then we can expect that solutions of (2.1) (or equivalently (4.1)) to collapse. However, when $\psi_{t} \leq 0$, we can have blowing up solutions. We only had to impose the sign condition on $\psi_{t}=P$ in (2.1) because $P \geq 0$ in our simple model. However, now we do not have the sign requirement on $\psi_{t}$ since here $\psi_{t} \approx \mu-P$. The operator $\mathfrak{L}$ is of mixed type for arbitrarily small positive $W$.

In contrast to the discriminant for (5.13) we now have as the discriminant the quantity

$$
(D c)^{2} \psi_{x}^{2}+4 D c \psi_{t}
$$

We conclude from this that when $\beta=0$ we can expect an "elliptic" singularity to try to form near $\psi_{x}=0$ if $\psi_{t} \approx \mu-P<0$ while we expect "hyperbolic" collapse to occur near $\psi_{x}=0$ if $\psi_{t} \approx \mu-P>0$. This is precisely the situation for the initial data taken in (OS). One has a unique local positive maximum of $P-\mu$ at the center of the interval and a local negative minimum of $P-\mu$ at the ends of the interval.

In the case that $W \approx \gamma$, a computation similar to that leading to equation (5.13) leads us to the following approximating equation (where now $\psi=-\ln W$ )

$$
\mathfrak{L} \psi=\psi_{t t}-D\left(c_{2}-2\right) \psi_{x} \psi_{x t}-D\left(\left(1-c_{1}\right)\left(\mu-\psi_{t}\right)\right) \psi_{x x}=D \psi_{x x t} .
$$

where now for $n=1,2$

$$
c_{n}=\frac{n}{1+\nu \gamma}+\frac{\delta}{2 \gamma}
$$

This equation also has exact solutions. If we write $\psi=\mu t+v$ then we see that $\mu-\psi_{t}=-v_{t} \approx P /(1+\nu \gamma)$. Thus, if $1-c_{1}>0$, the exact solutions will tend to collapse for $P>0$ while if $1-c_{1}<0$, we should expect further attempt at singularity formation as then (5.15) will be of mixed type for positive $P$. We have

$$
1-c_{1}=\frac{\nu \gamma}{1+\nu \gamma}-\frac{\delta}{2 \gamma}
$$

Numerical experiment bears this out.

Finally, we turn to the case $W>>$. In order to see more precisely what happens when $W$ is large, we use the approximations

$$
\begin{aligned}
A & \approx \frac{1}{\nu} \\
\frac{A^{\prime}(W)}{A(W)} & \approx \frac{1}{\nu W^{2}} \\
\frac{\Phi^{\prime}(W)}{\Phi(W)} & \approx \frac{\delta}{W^{2}}
\end{aligned}
$$


and set

$$
m_{i}=\frac{i}{\nu}+\delta \text { for } i=1,2 .
$$

Then our approximating equation for $W$ becomes

$$
\begin{aligned}
W_{t t}+m_{2} D \frac{W_{x}}{W^{2}} W_{x t}+ & D\left(m_{1} \frac{W_{t}}{W^{2}}-\mu\right) W_{x x} \\
= & D W_{x x t}+\left(\frac{W_{t}}{W^{2}}-\mu\right) W_{t}-m_{2} \mu D\left(\frac{W_{t}}{W^{2}}+\frac{\mu}{W}\right) W_{x}^{2}
\end{aligned}
$$

We now set $Z=1 / W,(\xi, \eta)=-\nabla W / W=\nabla Z / Z$. For $Z$ small

$$
P \approx \nu\left(W_{t}+\mu W\right)=\frac{\nu \mu}{Z}-\frac{\nu Z_{t}}{Z^{2}}
$$

so that

$$
\begin{aligned}
\mathfrak{L} Z & \equiv Z_{t t}-4 D \xi Z_{x t}-D\left[\left(m_{2}-2\right) \eta Z+\mu\right] Z_{x x} \\
& =D Z_{x x t}+\left[D m_{2} \xi^{2}(\eta-\mu)-\mu \eta\right] Z+\left(6 D \xi^{2} \eta-m_{t} \eta^{2}\right) Z
\end{aligned}
$$

which becomes, upon neglecting the quadratic and higher order terms in the variables $Z, \eta, \xi$,

$$
\mathfrak{L} Z \equiv Z_{t t}-4 D \xi Z_{x t}-D \mu Z_{x x}=D Z_{x x t}
$$

for which $B(Q) \geq 4 D \mu>0$. Thus, when $Z$ and its relative gradient are small, we can expect hyperbolic collapse as the characteristics have slopes of opposite sign. The mechanism for this is easily understood if we neglect the mixed second derivative term in (5.18). Then we have

$$
\mathfrak{L} Z \equiv Z_{t t}-D \mu Z_{x x}=D Z_{x x t}
$$

A short calculation with Fourier series shows us that every solution of this equation which is square integrable in a half strip $[a, b] \times(0, \infty)$ must be the sum of a linear function of $t$ plus a term which decays exponentially fast. Since $Z$ is to be small, this linear function must be constant. This suggests that if $W$ reaches a large maximum value at $\left(\ell / 2, t_{0}\right)$, say, we can expect that $P \approx \mu(1+\nu W)$ to decrease for times $t>t_{0}$ and to propagate away from the line of symmetry $x=\ell / 2$. We expect hyperbolic collapse of $P$ to a (large) constant value.

In order to summarize the preceeding discussion more concisely, it is helpful to think of (5.10) rewritten in terms of the relative gradient. However, as remarked above, that is a rather messy equation. Therefore we shall content ourselves with a discussion based upon the approximate form of (5.10) (which actually contains $(5.13),(5.14),(5.15)$ and $(5.18)$ as special cases $)$ :

$$
\mathfrak{L} \psi=\psi_{t t}-D\left(M_{2}(W)-2\right) \psi_{x} \psi_{x t}-D\left(\left(1-M_{1}(W)\right)\left(\mu-\psi_{t}\right)\right) \psi_{x x}=D \psi_{x x t} .
$$

In Figure 15 we have indicated how the characteristics might fit together in the physical plane based on the observations of this section. 
Figure 16. Schematic sketch of the characteristics in a generic case such as Figure 13.3. The regions 1,2,3 correspond to the regions so numbered in Figure 9. The nodal lines for $E, E_{1}$ should coaless at infinity in the continuous problem. In each of the regions 1 the slopes of the characteristics are of the same sign. We expect that the nodal lines for $E, E 1$ coaless to produce the "walls" observed by Othmer and Stevens but infinity, rather than in finite time. This is only supposition, however.

VI Remarks on numerics. We used a very simple explicit finite difference marching scheme to compute the various Matlab generated figures below. Because of this, we have used a time scale for our problem which is shorter than that of Othmer and Stevens (Figure 10) by a factor of 10. In spite of this, we found that for rather small values of $W(x, 0)$, (Figures 17.n, 18) that the numerically computed values of $P(x, t)$ become negative. This is a contradiction of the maximum principle. The reason for this apparent contradiction lies in the fact that the nonlinear analog the Courant-Lewy-Friedrichs condition, which is now a solution dependent condition, breaks down in finite time. Numerical experiments show that this breakdown occurs somewhat before $P$ becomes negative. For example, in Figure 17.1 it was found that the CFL condition fails at around $t=0.83$. Further experimentation shows that as we let $\Delta x \rightarrow 0$ in such a way that $\Delta t /\left(D(\Delta) x^{2}<0.5\right.$, these negative "islands" recede off to infinity. (Inspection of Figures 1.n in reverse order shows 
this experimentally.) (The nodal line for $P$ is offset from that for $E$ in Figures 17 and Figure 18 for the following reason: Since the Matlab program generates $P$ over a grid of size $N$ in the $x$ direction, then it generates $B$ over a grid of size $N-2$ in the $x$ direction because it uses central differences to evaluate $\xi=-W_{x} / W$. Notice in Figures 17.n that as the step size $\Delta x=h$ decreases, the level sets of $E$ and $P$ coalesce as the nodal line for $P$ moves off. This little apparent programming glitch is a useful visual aid!)

Figure 17.1. Same data as in Figure 13.4 but for a longer time. Here $\Delta x=h=0.005, \delta t=0.002$. 
Figure 17.2. Same data as in Figure 13.4. Here $\Delta x=h=0.006667, \delta t=$ 0.002 .

Figure 17.3. Same data as in Figure 13.4. Here $\Delta x=h=0.01, \delta t=$ 0.002 . 
Figure 18. Same data as in Figure 13.5. "Beyond blowup". The numerical scheme develops an instability which is manifested in the negative values that $P(x, t)$ assumes near the "blow up point".

\section{REFERENCES}

[A] W. Alt, Biased random walk models for chemotaxis and related diffusion approximations, J. Math. Biol. 9 (1980), 147-177.

[BGHP] Bertsch, M., Gurtin, M. E., Hilhorst,D. and Peletier, B., On interacting populations that disperse to avoid crowding: The effect of a sedentary colony, J. Math. Biol. 19 (1984), 1-12.

[CP] S. Childres and J. K. Percus, Nonlinear aspects of chemotaxis, Math. Biosciences 56 (1981), 217-237.

[D] B. Davis, Reinforced Random Walks, Prob. Th. Rel. Fields 84 (1990), 203-229.

[JL] W. Jager and S. Luckhaus, On explosions of solutions to a system of partial differential equations modelling chemotaxis, Trans. Amer. Math. Soc. 329 (1992), 819-824.

[KS] E. F. Keller and L.A. Segel, Initiation of slime mold aggregation viewed as an instability, J. Theoret. Biol. 26 (1970), 399-415.

[N] V. Nanjudiah, Chemotaxis, signal relaying and aggregation morphology, J. Theoret. Biol. 42 (1983), 63.

[OS] H. G. Othmer and A. Stevens, Aggregation, blowup and collapse: the ABCs of taxis and reinforced random walks, Preprint.

[ODA] H. G. Othmer, S. R. Dunbar and W. Alt, Models of dispersal in biological systems, J. Math. Biol. 26 (1988), 263-298.

[P] C. S. Patlak, Random walk with persistence and external bias, Bull. Math. 
Biophy. 15 (1953), 311-338.

[S] R. Schaaf, Stationary solutions of chemotaxis systems, Trans. Amer. Math. Soc. 292 (1985), 531-556. 